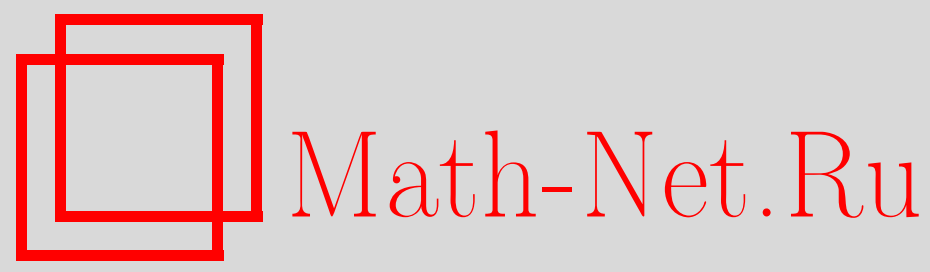

Н. В. Артамонов, Об устойчивости решений одного уравнения, возникающего в гидромеханике, Матем. заметки, 2000, том 67, выпуск 1, 15-24

DOI: https://doi.org/10.4213/mzm809

Использование Общероссийского математического портала Math-Net.Ru подразумевает, что вы прочитали и согласны с пользовательским соглашением http://www . mathnet.ru/rus/agreement

Параметры загрузки:

IP: 54.224 .187 .69

26 апреля 2023 г., $12: 15: 37$

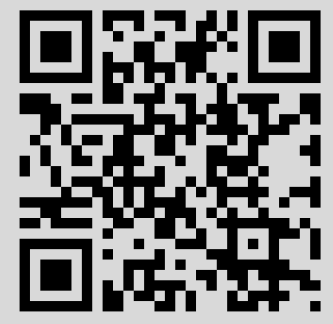




\section{ОБ УСТОЙЧИВОСТИ РЕШЕНИЙ ОДНОГО УРАВНЕНИЯ, ВОЗНИКАЮЩЕГО В ГИДРОМЕХАНИКЕ}

\section{Н. В. Артамонов}

В работе рассматривается вопрос о сильной устойчивости уравнения малых колебаний упругого трубопровода, по которому течет идеальная жидкость. Доказана теорема о существовании и единственности обобщенного решения и найдены достаточные условия сильной устойчивости в случае пульсирующей жидкости.

Библиограффия: 9 названий.

1. Введение. Уравнение мальх поперечных колебаний шарнирно закрепленного прямолинейного трубопровода, по которому течет идеальная жидкость со скоростью $v(t)$ и при отсутствии внутреннего и внешнего трения, имеет вид [1] (в безразмерных координатах):

$$
\begin{gathered}
u_{t t}+2 \beta v(t) u_{t x}+u_{x x x x}+v^{2}(t) u_{x x}+\beta v^{\prime}(t) u_{x}=0 \\
0 \leq x \leq 1, \quad t \geq 0, \quad \beta \geq 0 \\
\left.u\right|_{x=0}=\left.u\right|_{x=1}=\left.u_{x x}\right|_{x=0}=\left.u_{x x}\right|_{x=1}=0 \\
u(0, x)=u_{0}(x), \quad u_{t}(0, x)=u_{1}(x)
\end{gathered}
$$

Одним из основных является вопрос об устойчивости уравнения (1). В стационарном случае $\left(v(t) \equiv v_{0}\right)$ исследование устойчивости сводится к изучению спектральных свойств квадратичного пучка, получающегося при разделении переменных. В работах [2], [3] достаточно полно исследованы пучки более общего вида. Случай стационарного уравнения с учетом трения подробно исследован в [3], [4]: при $v_{0}<\pi$ спектр квадратичного пучка лежит в левой полуплоскости (уравнение устойчиво), при $v_{0} \geq \pi$ уравнение неустойчиво. Нестационарное уравнение с учетом трения изучалось в [5] методом функций Ляпунова в предположении, что скорость имеет вид $v(t)=v_{0}+\varepsilon \cos (\omega t)$. Показано, что при $0 \leq v_{0}<\pi$ будет устойчивость при достаточно малых $\varepsilon$ и при любых $\omega>0$, при $v_{0} \geq \pi$ устойчивости нет. В данной работе функционально-аналитическими методами изучается устойчивость уравнения (1) в случае, когда скорость имеет вид

Работа выполнена при поддержке Российского фонда фундаментальных исследований, грант № 99-01-01079. 
$v(t)=v_{0}+\varepsilon \tilde{v}(t)$, где $\varepsilon-$ мало и $\tilde{v}(t)-T$-периодическая функция. Так как спектр невозмущенного уравнения лежит на мнимой оси (за исключением конечного числа собственных значений), то использование метода функций Ляпунова является неэффективным. В работе применяется другой подход, основанньй на сведении уравнения (1) к линейному гамильтоновому операторно-дифференциальному уравнению в сепарабельном гильбертовом пространстве и применении теории операторов в пространствах с индефинитной метрикой.

2. Существование и единственность решения. Рассмотрим вопрос о существовании и единственности решения уравнения. Введем сепарабельное гильбертово пространство $\mathcal{H}=L_{2}[0,1]$ и операторы в $\mathcal{H}:$

$$
\begin{aligned}
& P_{0}: D\left(P_{0}\right)=\left\{y \in W_{2}^{4}(0,1) \mid y(0)=y(1)=y^{\prime \prime}(0)=y^{\prime \prime}(1)=0\right\}, \quad P_{0} y=\frac{d^{4} y}{d x^{4}} \\
& P_{1}: D\left(P_{1}\right)=\left\{y \in W_{2}^{2}(0,1) \mid y(0)=y(1)=0\right\}, \quad P_{1} y=\frac{d^{2} y}{d x^{2}} \\
& B: D(B)=D\left(P_{1}\right), \quad B y=\beta \frac{d y}{d x} .
\end{aligned}
$$

Они обладают следующими свойствами:

(А) $P_{0}$ - самосопряженный положительно определенньй оператор, $P_{0}^{-1} \in \mathfrak{S}_{\infty}$; через $\mathcal{H}_{\theta}$ будем обозначать шкалу гильбертовых пространств, построенную по оператору $P_{0}[6]$;

(В) $P_{1}-$ замкнутьй симметрический оператор, $D\left(P_{1}\right) \supset D\left(P_{0}^{\frac{1}{2}}\right)$;

(C) $B$ - замкнутьй кососимметрический оператор, $D(B) \supset D\left(P_{0}^{\frac{1}{2}}\right)$.

Уравнение (1) с краевыми условиями (2) можно записать в виде операторно-дифференциального уравнения в $\mathcal{H}$ :

$$
u^{\prime \prime}+2 v(t) B u^{\prime}+P_{0} u+v^{2}(t) P_{1} u+v^{\prime}(t) B u=0
$$

с начальньми условиями

$$
u(0)=u_{0}, \quad u^{\prime}(0)=u_{1}
$$

Для дальнейших рассуждений конкретный вид операторов $P_{0}, P_{1}, B$ не существенен, важны лишь свойства (A), (B), (C). Отметим, что по теореме об интерполящии [6] из (В), (C) следует, что $P_{1} \in \mathfrak{B}\left(\mathcal{H}_{\theta}, \mathcal{H}_{\theta-\frac{1}{2}}\right)$ и $B \in \mathfrak{B}\left(\mathcal{H}_{\theta}, \mathcal{H}_{\theta-\frac{1}{2}}\right), 0 \leq \theta \leq 1 / 2$. Дадим определения классического и обобщенного решения уравнения (3).

ОПРЕДЕЛЕНИЕ 1. Функция $u(t) \in \mathcal{H}_{1}, a \leq t \leq b$, дважды сильно непрерьвно дифференцируемая в $\mathcal{H}$, назьвается классическим решением уравнения $(3)$, если $u^{\prime}(t) \in \mathcal{H}_{\frac{1}{2}}$, $u(t)$ сильно дифференцируема в $\mathcal{H}_{\frac{1}{2}}$ и удовлетворяет уравнению $(3)$ на $[a, b]$ в пространстве $\mathcal{H}$. 
ОПРЕДЕЛЕНИЕ 2. Функция $u(t) \in \mathcal{H}_{\frac{1}{2}}, a \leq t \leq b$, называется обобщенны.м решением уравнения (3), если

1) $u(t)$ абсолютно непрерывна на $[a, b]$ в пространстве $\mathcal{H}, u^{\prime}(t)$ слабо непрерьвна на $[a, b]$ в $\mathcal{H}, u^{\prime}(t)$ абсолютно непрерывна на $[a, b]$ в пространстве $\mathcal{H}_{-\frac{1}{2}}$;

2) при п.в. $t \in[a, b] u(t)$ удовлетворяет уравнению

$$
\left(u^{\prime}+v(t) B u\right)^{\prime}+P_{0} u+v^{2}(t) P_{1} u+v(t) B u^{\prime}=0
$$

в пространстве $\mathcal{H}_{-\frac{1}{2}}$.

TeOpema 1. Пусть $a>0 u v(t) \in C^{1}[0, a]$. Тогдa

а) если $u_{0} \in \mathcal{H}_{1}, u_{1} \in \mathcal{H}_{\frac{1}{2}}$, то существует единственное классическое решение на [0, а] с начальными условиями (4);

b) если $u_{0} \in \mathcal{H}_{\frac{1}{2}}, u_{1} \in \mathcal{H}$, то существует единственное обобщенное решение на $[0, a]$ с начальными условиями (4).

ДокАЗАтЕльство. а) Существование классического решения было доказано в [5].

b) Определим следующие операторы, заданные в виде матриц:

$$
\begin{aligned}
& H_{0}=\left(\begin{array}{cc}
P_{0} & 0 \\
0 & I
\end{array}\right), \quad \mathcal{J}=\left(\begin{array}{cc}
0 & -I \\
I & 0
\end{array}\right), \quad F=i H_{0}^{-\frac{1}{2}} J H_{0}^{-\frac{1}{2}}, \\
& G_{1}(t)=\left(\begin{array}{cc}
-v^{2}\left(P_{0}^{-\frac{1}{2}} B\right)\left(B P_{0}^{-\frac{1}{2}}\right) & v\left(P_{0}^{-\frac{1}{2}} B\right) \\
-v B P_{0}^{-\frac{1}{2}} & 0
\end{array}\right), \quad G_{2}(t)=\left(\begin{array}{cc}
v^{2} P_{0}^{-\frac{1}{2}} P_{1} P_{0}^{-\frac{1}{2}} & 0 \\
0 & 0
\end{array}\right),
\end{aligned}
$$

$H_{0}$ - самосопряженньй, положительно определенный оператор в $\mathcal{H} \times \mathcal{H}$ с областью определения $D\left(H_{0}\right)=\mathcal{H}_{1} \times \mathcal{H} ; \mathcal{J}, F, G_{1}(t)$ и $G_{2}(t)$ - ограниченные операторы в $\mathcal{H} \times \mathcal{H}$, причем $G_{1}^{*}(t)=G_{1}(t), G_{2}^{*}(t)=G_{2}(t), F^{*}=F, \mathcal{J}^{*}=-\mathcal{J}$. Перейдем от уравнения 2-го порядка к уравнению первого порядка в пространстве $X=\mathcal{H} \times \mathcal{H}_{-\frac{1}{2}}$. Пусть $u(t)-$ обобшенное решение. Положим

$$
w_{1}(t)=u(t), \quad w_{2}(t)=u^{\prime}(t)+v(t) B u(t) .
$$

Тогда $w(t)=\left(w_{1}(t), w_{2}(t)\right) \in \mathcal{H}_{\frac{1}{2}} \times \mathcal{H}$ удовлетворяет в пространстве $X$ уравнению

$$
w^{\prime}=\mathcal{J}^{-1} H_{0}^{\frac{1}{2}}\left(I+G_{1}(t)+G_{2}(t)\right) H_{0}^{\frac{1}{2}} w
$$

Обозначим $A(t) \equiv \mathcal{J}^{-1} H_{0}^{\frac{1}{2}}\left(I+G_{1}(t)+G_{2}(t)\right) H_{0}^{\frac{1}{2}}, D(A(t))=\mathcal{H}_{\frac{1}{2}} \times \mathcal{H}$ и рассмотрим пространство $Y=\mathcal{H}_{\frac{1}{2}} \times \mathcal{H} . Y$ является гильбертовым пространством и, следовательно, рефлексивным, оно плотно вложено в $X,\|f\|_{X} \leq$ const $\|f\|_{Y} \forall f \in Y$. Так как $H_{0}^{\frac{1}{2}} \in \mathfrak{B}(Y, \mathcal{H} \times \mathcal{H})$ и $\mathcal{J}^{-1} H_{0}^{\frac{1}{2}} \in \mathfrak{B}(\mathcal{H} \times \mathcal{H}, X)$, то $A(t) \in \mathfrak{B}(Y, X) \forall t \in[0, a]$, $\|A(t)\|_{Y, X} \in C[0, a]$, и отображение $F: X \rightarrow Y$ является изоморфизмом банаховых пространств. Положим

$$
S(t)=\left(\begin{array}{cc}
-v(t) P_{0}^{-\frac{1}{2}} B & P_{0}^{-\frac{1}{2}} \\
P_{0}^{\frac{1}{2}} & 0
\end{array}\right) \in \mathfrak{B}(X)
$$


ограниченно обратимый оператор, $S(t) Y=Y$. Так как $v(t) \in C^{1}[0, a]$, то $S(t)$ - оператор-функция ограниченной вариации (относительно нормы $\mathfrak{B}(X)$ ) и $\|S(t)\| \leq$ const на $[0, a]$. Имеем

$$
S(t) \mathcal{J}^{-1} H_{0}^{\frac{1}{2}}\left(I+G_{1}(t)\right) H_{0}^{\frac{1}{2}} S^{-1}(t)=A_{1}(t) \equiv\left(\begin{array}{cc}
0 & I_{1} \\
-P_{0} & -2 v(t) P_{0}^{\frac{1}{2}} B P_{0}^{-\frac{1}{2}}
\end{array}\right) .
$$

Так как $i A_{1}(t)$ - самосопряженный оператор в $X$, для любого $n \in \mathbb{N}$ и для произвольных $n$ точек $0 \leq t_{1} \leq \ldots \leq t_{n} \leq a$ верно неравенство

$$
\left\|\prod_{j=1}^{n}\left(A_{1}\left(t_{j}\right)-\lambda I\right)^{-1}\right\|_{X} \leq \lambda^{-n} \quad \forall \lambda>0 .
$$

Отметим, что $\mathcal{J}^{-1} H_{0}^{\frac{1}{2}} G_{2}(t) H_{0}^{\frac{1}{2}} \in \mathfrak{B}(X)$. Применяя предложения 3.5 и 4.4 из работы [7], получаем, что существуют числа $M, C>0$ такие, что $\forall n \in \mathbb{N}$ и произвольных $n$ точек $0 \leq t_{1} \leq \ldots \leq t_{n} \leq a$ справедлива оценка

$$
\left\|\prod_{j=1}^{n}\left(A\left(t_{j}\right)-\lambda I\right)^{-1}\right\|_{X} \leq M(\lambda-\mathrm{C})^{-n} \forall \lambda>C .
$$

Аналогично показьвается, что для оператора $F^{-1} A(t) F$ имеет место похожая оценка: сушествуют числа $M_{1}, C_{1}>0$ такие, что при $\lambda>C_{1} \forall n \in \mathbb{N}, 0 \leq t_{1} \leq \cdots \leq t_{n} \leq a$

$$
\left\|\prod_{j=1}^{n}\left(F^{-1} A(t) F-\lambda I\right)^{-1}\right\|_{X} \leq M_{1}\left(\lambda-C_{1}\right)^{-n} \text {. }
$$

Применяя теоремы 4.1 и 5.1 из работы [7], получаем, что существует единственное семейство операторов $U(t, s), 0 \leq s \leq t \leq a$, такое, что:

i) $U(t, s)$ - сильно непрерьвно на $X, U(s, s)=I$ и

$$
\|U(t, s)\|_{X} \leq M \exp \{C(t-s)\}
$$

ii) $U(t, s)=U(t, r) U(r, s), s \leq r \leq t$ (групповое свойство);

iii) $U(t, s) Y \subset Y, U(t, s)$ слабо непрерьвно по $t, s$ в $Y$ и

$$
\|U(t, s)\|_{Y} \leq M_{1} \exp \left\{C_{1}(t-s)\right\} ;
$$

iv) $\forall y_{0} \in Y$ при $t \in[s, a]$ функция $U(t, s) y_{0}$ в $X$ абсолютно непрерывна, сильно дифференцируема, удовлетворяет уравнению (6) и производная слабо непрерьвна.

Пусть $u_{0} \in \mathcal{H}_{\frac{1}{2}}, \quad u_{1} \in \mathcal{H}$ - начальные данные (4). Положим $w_{0}=\left(u_{0}, u_{1}+\right.$ $\left.v(0) B u_{0}\right) \in Y$. Тогда существует единственное решение $w(t)=U(t, 0) w_{0}$ уравнения $(6)$ с начальньми данными $w(0)=w_{0}$. Из свойств i)-iv) получаем, что функция $u(t) \equiv$ $w_{1}(t)$ удовлетворяет свойствам 1$), 2$ ) определения 2. Таким образом, уравнение (3) корректно разрешимо в пространстве $\mathcal{H}_{\frac{1}{2}} \times \mathcal{H}$. Отметим также, что из результатов [7] следует, что разрешаюший оператор $U(t, s)$ непрерывно зависит от $\max _{[0, a]}\|A(t)\|_{Y, X}$.

Если рассмотреть уравнение (4) с “обратньп" временем $\tau=-t$, то для него также будут вьполнены условия теорем 4.1 и 5.1 из [7], откуда легко получить, что оператор $U(t) \equiv U(t, 0)$ обратим $\forall t \in[0, a]$. 
СлЕДСтвИЕ 1. Если $v(t) \in C^{1}[0, \infty)$, то решение $u(t)$ (классическое или обобщенное) определено на полуоси $[0, \infty)$.

ЗАмечАниЕ 1 . Так как $H_{0}^{\frac{1}{2}}(I+G(t)) H_{0}^{\frac{1}{2}}-$ симметрический (неограниченньй) оператор в пространстве $\mathcal{H} \times \mathcal{H}$, уравнение $(6)$ имеет вид гамильтоновой системы в $\mathcal{H} \times \mathcal{H}$ [8]. Функция $w(t)$, определенная по формулам (5) в пространстве $\mathcal{H} \times \mathcal{H}$, удовлетворяет уравнению

$$
\left(H_{0}^{-\frac{1}{2}} \mathcal{J} w\right)^{\prime}=\left(I+G_{1}(t)+G_{2}(t)\right) H_{0}^{\frac{1}{2}} w,
$$

т.е. является обобщенным решением гамильтоновой системы [8].

Рассмотрим в $X$ оператор $\left|\mathcal{J}^{-1} H_{0}\right|, D\left(\left|\mathcal{J}^{-1} H_{0}\right|\right)=\mathcal{H}_{\frac{1}{2}} \times \mathcal{H}$, и построим по нему шкалу пространств $X_{\theta}, 0 \leq \theta \leq 1$. Легко проверить, что $X_{\theta}=\mathcal{H}_{\frac{\theta}{2}} \times \mathcal{H}_{\frac{\theta-1}{2}}$ и $X_{1}=Y$. Так как $U(t) \in \mathfrak{B}\left(X_{1}, X_{1}\right)$ и $U(t) \in \mathfrak{B}\left(X_{0}, X_{0}\right)$, по теореме об интерполяции [6] $U(t) \in$ $\mathfrak{B}\left(X_{\theta}, X_{\theta}\right), 0 \leq \theta \leq 1$. Для любых двух решений $w(t), \widetilde{w}(t)$ уравнения (7) вьполнено соотношение [8]:

$$
(\mathcal{J} w(t), \widetilde{w}(t))_{\mathcal{H} \times \mathcal{H}}=\text { const } .
$$

Так как $\left|\mathcal{J}^{-1} H_{0}\right|^{-\theta} \in \mathfrak{B}\left(X, X_{\theta}\right)$ и $\left|H_{0}^{-\frac{1}{2}} \mathcal{J}\right| \in \mathfrak{B}(X, \mathcal{H} \times \mathcal{H})$ - изометрические операторы, то $\left(S_{\theta}=\left|\mathcal{J}^{-1} H_{0}\right|^{-\theta}\left|H_{0}^{-\frac{1}{2}} \mathcal{J}\right|^{-1} \in \mathfrak{B}\left(\mathcal{H} \times \mathcal{H}, X_{\theta}\right)\right)$

$$
\left(S_{\theta} \mathcal{J} w(t), S_{\theta} \widetilde{w}(t)\right)_{\theta}=\text { const, }
$$

где $(\cdot, \cdot)_{\theta}$ - скалярное произведение в $X_{\theta}$ (норму в $X_{\theta}$ будем обозначать через $\left.\|\cdot\|_{\theta}\right)$. При $\theta=1$ получим

$$
(\widehat{F} w(t), \widetilde{w}(t))_{1}=\mathrm{const}, \quad \widehat{F}=i S_{1}^{2} \mathcal{J} \in \mathfrak{B}\left(X_{1}\right), \quad \widehat{F}^{*}=\widehat{F},
$$

откуда следует (в силу произвольности $w(t)$ и $\widetilde{w}(t))$, что $U^{*}(t) \widehat{F} U(t)=\widehat{F}$. Так как $U^{-1}(t) \in \mathfrak{B}\left(X_{1}\right)$, оператор $U(t)$ является $\widehat{F}$-унитарным в пространстве $X_{1}$ [9] (отметим, что оператор $\widehat{F}^{-1}$ может быть неограниченным). При $\theta=1 / 2$ получаем

$$
(\widehat{\mathcal{J}} w(t), \widetilde{w}(t))_{\frac{1}{2}}=\text { const }, \quad \widehat{\mathcal{J}}=i S_{\frac{1}{2}}^{2} \mathcal{J} \in \mathfrak{B}\left(X_{\frac{1}{2}}\right)
$$

- ограниченно обратимый оператор и $\widehat{\mathcal{J}}^{*}=-\widehat{\mathcal{J}}$. В силу произвольности $w(t), \widetilde{w}(t)$ получаем, что $U^{*}(t) \widehat{\mathcal{J}} U(t)=\widehat{\mathcal{J}}$. Так как $U^{-1}(t) \in \mathfrak{B}\left(X_{\frac{1}{2}}\right)$, то оператор $U(t)$ является $\widehat{\mathcal{J}}$-унитарным в пространстве $X_{\frac{1}{2}}[9]$.

3. Исследование устойчивости уравнения (3). Следуя [9], будем говорить,что уравнение (6) устойчиво, если все решения ограничены в пространстве $X_{\frac{1}{2}}$, т.е. для любого его решения вьполнено неравенство

$$
\|w(t)\|_{\frac{1}{2}} \leq C\|w(0)\|_{\frac{1}{2}}
$$

с константой, не зависящей от решения (в [9] условие записьвалось в другой форме). Если функция $v(t)$ ограничена на $\mathbb{R}_{+}$, то это неравенство эквивалентно следующему:

$$
\left\{\|u(t)\|_{\frac{1}{4}, \mathcal{H}}^{2}+\left\|u^{\prime}(t)\right\|_{-\frac{1}{4}, \mathcal{H}}^{2}\right\} \leq \mathrm{C}\left\{\|u(0)\|_{\frac{1}{4}, \mathcal{H}}^{2}+\left\|u^{\prime}(0)\right\|_{-\frac{1}{4}, \mathcal{H}}^{2}\right\}, \quad t \in[0, \infty),
$$

где $\|\cdot\|_{\theta, \mathcal{H}}-$ норма в пространстве $\mathcal{H}_{\theta}$. 
ОПРЕДЕЛЕНИЕ 3. Уравнение (3) назьвается устойчивым, если для любого его обобщенного решения вьполнена оценка (9) с константой, не зависящей от решения $u(t)$.

Далее будем рассматривать случай, когда скорость $v(t)$ имеет следующий вид: $v(t)=$ $v_{0}+\varepsilon \tilde{v}(t)$, где $\varepsilon \geq 0$ и $\tilde{v}(t) \in C^{1}[0, \infty)-T$-периодическая функция.

ОПРЕДЕЛЕНИЕ 4. Уравнение (3) назьвается сильно устойчивым, если существует $\varepsilon_{0}>0$ такое, что при $0 \leq \varepsilon \leq \varepsilon_{0}$ уравнение устойчиво.

В силу $T$-периодичности функции $v(t)$ имеем $U(t)=U(\tau) U^{n}(T)$, где $t=\tau+n T$, $0 \leq \tau<T$, откуда следует, что неравенство (8) эквивалентно следующему:

$$
\left\|U^{n}(T)\right\|_{\frac{1}{2}} \leq \text { const, } \quad n=0, \pm 1, \pm 2, \ldots
$$

Следуя [9], будем называть $\widehat{\mathcal{J}}$-унитарньй оператор $U$ устойчивылм, если для него выполнено неравенство (10), и сильно устойчивым, если существует $c>0$ такое, что при выполнении неравенства $\|\widetilde{U}-U\|_{\frac{1}{2}}<c$ все $\widehat{\mathcal{J}}$-унитарные операторы устойчивы. Если ввести для семейства $\{A(t)\}$ норму

$$
\|A\|=\max _{[0, T]}\|A(t)\|_{Y, X}=\max _{[0, T]}\left\|I+G_{1}(t)+G_{2}(t)\right\|_{\mathcal{H} \times \mathcal{H}},
$$

то малым изменениям семейства $\{A(t)\}$ в смысле этой нормы отвечают малые изменения $U(T)$ в смысле равномерной операторной нормы. Обозначим через $U_{\varepsilon}(T)$ оператор $U(T)$ в случае, когда скорость имеет вид $v(t)=v_{0}+\varepsilon \tilde{v}(t)$. Так как норма $\{A(t)\}$ непрерьвно зависит от $\varepsilon$, то отображение $\varepsilon \rightarrow U_{\varepsilon}(T)$ непрерывно и получаем

ПРЕДЛОЖЕНИЕ 1. Для того чтобы уравнение (3) было сильно устойчиво, достаточно, чтобы оператор $U_{0}(T)$ был сильно устойчив.

При $\varepsilon=0$ уравнение (3) имеет постоянные коэффициенты; пусть $L(\lambda)=\lambda^{2} I+$ $2 v_{0} \lambda B+P_{0}+v_{0}^{2} P_{1}-$ пучок, получающийся при разделении переменных. Его спектр $\sigma(L)$ дискретен (в силу условия $(\mathrm{A}))$, симметричен относительно мнимой оси и если оператор $P_{0}+v_{0}^{2} P_{1}$ положительно определен, то спектр чисто мнимый и алгебраически простой [2]. В уравнении (6) $A \equiv A(t)$ постоянный оператор, $\sigma_{d}(A)=\sigma(L)$ и $g_{n}=$ $\left(e_{n}, \lambda_{n} e_{n}+B e_{n}\right) \in X_{1}-$ собственньй вектор, отвечающий собственному значению $\lambda_{n}$ $\left(e_{n} \in \operatorname{Ker} L\left(\lambda_{n}\right)\right)$. Тогда $U(t) g_{n}=e^{\lambda_{n} t} g_{n}$. Если существует собственное значение (с.з.) пучка $L$, не лежащее на мнимой оси, или присоединенньй вектор, то уравнение неустойчиво; поэтому далее считаем, что спектр алгебраически простой и целиком лежит на мнимой оси. Отметим, что если $0 \in \sigma(L)$, то существует присоединенньй вектор, отвечающий с.з. 0. Так как векторы $\left\{\left(e_{n}, \lambda_{n} e_{n}\right) \mid \lambda_{n} \in \sigma(L), e_{n} \in \operatorname{Ker} L\left(\lambda_{n}\right)\right\}$ образуют базис Рисса в пространстве $X_{1}=\mathcal{H}_{\frac{1}{2}} \times \mathcal{H}[2]$, то собственные векторы $\left\{g_{n}\right\}$ оператора $U(t)$ образуют базис Рисса в пространстве $X_{1}$ и, следовательно, в $X_{1}$

$$
\sigma\left(U_{0}(T)\right)=\overline{\left\{e^{\lambda T} \mid \lambda \in \sigma(L)\right\}}
$$


Обозначим $a(x, \lambda)=|\lambda|^{2}(x, x)+\left(P_{0} x+v_{0}^{2} P_{1} x, x\right), x \in \mathcal{H}_{1}$, и введем следующие множества:

$$
\begin{aligned}
& \sigma_{I}=\overline{\left\{e^{\lambda T} \mid \lambda \in \sigma(L), i \lambda^{-1} a(x, \lambda)>0, \quad \forall x \in \operatorname{Ker} L(\lambda)\right\}}, \\
& \sigma_{I I}=\overline{\left\{e^{\lambda T} \mid \lambda \in \sigma(L), i \lambda^{-1} a(x, \lambda)<0, \quad \forall x \in \operatorname{Ker} L(\lambda)\right\}}, \\
& \sigma_{0}=\left\{e^{\lambda T} \mid \lambda \in \sigma(L), \quad \exists x \in \operatorname{Ker} L(\lambda) \quad a(x, \lambda)=0\right\} .
\end{aligned}
$$

Тогда $\sigma\left(U_{0}(T)\right)=\sigma_{I} \cup \sigma_{I I} \cup \bar{\sigma}_{0}$. Отметим, что если оператор $P_{0}+v_{0}^{2} P_{1}$ положительно определен, то $\sigma_{0}=\varnothing$ и

$$
\sigma_{I}=\overline{\left\{e^{\lambda T} \mid \lambda \in \sigma(L), \quad \operatorname{Im} \lambda>0\right\}}, \quad \sigma_{I I}=\overline{\left\{e^{\lambda T} \mid \lambda \in \sigma(L), \operatorname{Im} \lambda<0\right\}}
$$

Пусть $\sigma \subset \sigma\left(U_{0}(T)\right)$ - изолированная часть спектра, $P_{\sigma}$ - проектор Рисса, $g_{n}-$ собственньй вектор, отвечающий с.з. $\nu_{n}=\exp \left\{\lambda_{n} T\right\}$ (с.з. считаем столько раз, какова его кратность). Тогда

$$
\mathfrak{L}_{\sigma}=P_{\sigma} X=\left\{\sum_{\nu_{n} \in \sigma} c_{n} g_{n} \mid\left\{c_{n}\right\}_{1}^{\infty} \in l_{2}\right\} .
$$

Так как $\left\{\left(e_{n}, \lambda_{n} e_{n}\right)\right\}$ - собственные векторы оператора, симметричного относительно индефинитной метрики [2]

$$
\begin{gathered}
{[x, y]=\left(P_{0} x_{1}+v_{0}^{2} P_{1} x_{1}, y_{1}\right)_{\mathcal{H}}+\left(x_{2}, y_{2}\right)_{\mathcal{H}},} \\
x=\left(x_{1}, x_{2}\right), \quad y=\left(y_{1}, y_{2}\right)
\end{gathered}
$$

они ортогональны относительно этой метрики. Отсюда, учитьвая что $L\left(\lambda_{n}\right) e_{n}=0$, легко получить, что $\left(\widehat{F} g_{n}, g_{m}\right)_{1}=0$ при $n \neq m$ и $(\widehat{F} f, f)_{1}=\sum\left|c_{k}\right|^{2}\left(\widehat{F} g_{k}, g_{k}\right)_{1} \forall f \in X_{1}$, $f=\sum c_{k} g_{k}$. Подпространство $\mathfrak{L} \subset X$ назовем положительным (отрииательным) относительно $\widehat{F}$, если $(\widehat{F} f, f)_{1}>0(<0) \forall f \in \mathfrak{L}, f \neq 0$. Так как $\left(\widehat{F} g_{k}, g_{k}\right)_{1}>0(<0)$, если $e^{\lambda_{k} T} \in \sigma_{I} \quad\left(e^{\lambda_{k} T} \in \sigma_{I I}\right)$, то подпространство $\mathfrak{L}_{\sigma}$ положительно (отрицательно) тогда и только тогда, когда $\sigma \subset \sigma_{I}\left(\sigma \subset \sigma_{I I}\right)$.

ТЕОрема 2. Пусть спектр пучка $L(\lambda)$ лежстт на мнимой оси и присоединенные векторы отсутствуют. Если $\sigma_{0}=\varnothing и \sigma_{I} \cap \sigma_{I I}=\varnothing$, то уравнение (3) сильно устойчиво.

ДокАЗАТЕЛЬСТВО. Покажем, что при вьполнении условий теоремы оператор $U_{0}(T)$ сильно устойчив. Так как $\sigma_{0}=\varnothing$, то $\sigma\left(U_{0}(T)\right)=\sigma_{I} \cup \sigma_{I I}$. Обозначим при $\psi<\theta$

$$
I(\psi, \theta)=\{z \in \mathbb{C}|| z \mid=1, \psi<\arg z<\theta\}
$$

- дуга единичной окружности. Пусть $z_{1} \in \sigma_{I}$. Тогда положим $I_{1} \equiv I\left(\psi_{1}, \theta_{1}\right)-$ наибольшая дуга единичной окружности такая, что $z_{1} \in I_{1}$ и $I_{1} \cap \sigma_{I I}=\varnothing$ (она существует, так как $\sigma_{I I}-$ замкнуто и $\left.\exp \left\{i \psi_{1}\right\}, \exp \left\{i \theta_{1}\right\} \in \sigma_{I I}\right)$. Если $\sigma_{I} \backslash I_{1} \neq \varnothing$, то пусть $z_{2} \in \sigma_{I} \backslash I_{1}$ и $I_{2} \equiv I\left(\psi_{2}, \theta_{2}\right)$ - наибольшая дуга такая, что $z_{2} \in I_{2}$ и $I_{2} \cap \sigma_{I I}=\varnothing$ $\left(\right.$ причем $\exp \left\{i \psi_{2}\right\}, \exp \left\{i \theta_{2}\right\} \in \sigma_{I I}$ в силу замкнутости $\sigma_{I I}$ и $\left.I_{1} \cap I_{2}=\varnothing\right)$. Если $\sigma_{I} \backslash$ 
$\left(I_{1} \cup I_{2}\right) \neq \varnothing$, то положим $z_{3} \in \sigma_{I} \backslash\left(I_{1} \cup I_{2}\right)$ и т.д. Если процесс продолжается неограничено, то получаем две последовательности $\left\{z_{n}\right\}_{1}^{\infty}$ и $\left\{\exp \left(i \psi_{n}\right)\right\}_{1}^{\infty}$ такие, что $\left|z_{n}-e^{i \psi_{n}}\right| \rightarrow 0(n \rightarrow \infty)$. Так как $\left\{z_{n}\right\}_{1}^{\infty} \subset \sigma_{I},\left\{\exp \left(i \psi_{n}\right)\right\}_{1}^{\infty} \subset \sigma_{I I}$ и $\sigma_{I}, \sigma_{I I}-$ замкнуты, получаем, что $\sigma_{I} \cap \sigma_{I I} \neq \varnothing$. Таким образом, существует конечный набор дуг $\left\{I_{k}\right\}_{k=1}^{N}$ :

$$
\sigma_{I} \subset \bigcup_{k=1}^{N} I_{k}, \quad \sigma_{I I} \subset\{z \in \mathbb{C}|| z \mid=1\} \backslash \bigcup_{k=1}^{N} I_{k},
$$

откуда следует, что

$$
\sigma_{I}=\bigcup_{k=1}^{N} \sigma(k), \quad \sigma_{I I}=\bigcup_{k=N+1}^{2 N} \sigma(k),
$$

где $\sigma(k)$ - изолированные части спектра. Подпространство $\mathfrak{L}_{k}=P_{\sigma(k)} X$ положительно, если $1 \leq k \leq N$, и отрицательно, если $N+1 \leq k \leq 2 N$. Таким образом, оператор $U_{0}(T)$ не имеет спектра смешанного рода в пространстве $X_{1}[9]$ и, следовательно, сильно устойчив [9]. Применяя предложение 1 , получаем, что уравнение (3) сильно устойчиво.

В случае уравнения (1) спектр пучка $L(\lambda)$ совпадает с собственными значениями краевой задачи

$$
\begin{gathered}
y^{I V}+v_{0}^{2} y^{\prime \prime}+2 \beta v_{0} \lambda y^{\prime}+\lambda^{2} y=0, \\
y(0)=y(1)=y^{\prime \prime}(0)=y^{\prime \prime}(1)=0 .
\end{gathered}
$$

При $v_{0}<\pi$ оператор $P_{0}+v_{0}^{2} P_{1}$ положительно определен и, следовательно, спектр задачи $(11),(12)$ чисто мнимьй. Положим $\lambda=i s^{2}$. Функция $e^{\mu x}$ является решением уравнения (11) тогда и только тогда, когда $\mu$-корень многочлена

$$
\left(\frac{\mu}{s}\right)^{4}+\frac{v_{0}^{2}}{s^{2}}\left(\frac{\mu}{s}\right)^{2}+2 i \beta \frac{v_{0}}{s}\left(\frac{\mu}{s}\right)-1=0 .
$$

При достаточно малом $v_{0} / s$ все корни многочлена различны и аналитически зависят от $v_{0} / s$. Вычисляя три первых коэффициента в ряде Тейлора для корней $\mu_{k}, k=$ $1, \ldots, 4$, получим

$$
\mu_{k}=s\left(\omega_{k}-\frac{1}{2} i \beta \omega_{k}^{2} \frac{v_{0}}{s}+\frac{1}{4 \omega_{k}}\left(1-\frac{1}{2} \beta^{2}\right) \frac{v_{0}^{2}}{s^{2}}+O\left(\frac{v_{0}^{3}}{s^{3}}\right)\right),
$$

где $\left\{\omega_{k}\right\}_{1}^{4}-$ корни из 1. Функции $y_{k}(x)=\exp \left\{\mu_{k} x\right\}$ образуют фундаментальную систему решений уравнения (11). Положим $y=\sum c_{k} y_{k}$ и, подставив $y(x)$ в краевые условия (12), получаем однородную систему линейных уравнений относительно $c_{k}$. Она имеет нетривиальное решение тогда и только тогда, когда $y(x)$ - собственная функция и $i s^{2}-$ собственное значение. Учитьвая, что $s^{2} \in \mathbb{R}$, после преобразований получим, что собственные значения краевой задачи должны удовлетворять одному из двух уравнений

$$
\begin{array}{r}
\sin s+\frac{1}{4 s}\left(1-\frac{1}{2} \beta^{2}\right) v_{0}^{2} \cos s+O\left(\frac{v_{0}^{3}}{s^{2}}\right)=0, \\
\sin i s+\frac{1}{4 i s}\left(1-\frac{1}{2} \beta^{2}\right) v_{0}^{2} \cos i s+O\left(\frac{v_{0}^{3}}{s^{2}}\right)=0 .
\end{array}
$$


Решая уравнения, получаем четыре последовательности

$$
s_{k, n}=\omega_{n}\left(\pi k-\left(1-\frac{1}{2} \beta^{2}\right) \frac{v_{0}^{2}}{4 \pi k}+O\left(\frac{v_{0}^{3}}{k^{2}}\right)\right), \quad k \in \mathbb{N}, \quad n=1, \ldots, 4
$$

Таким образом, собственные значения краевой задачи $(11),(12)$ имеют асимптотику

$$
\lambda_{k}= \pm i\left\{(\pi k)^{2}-\left(1-\frac{1}{2} \beta^{2}\right) \frac{v_{0}^{2}}{2}+O\left(\frac{v_{0}^{3}}{k}\right)\right\} .
$$

ПРЕДЛОЖЕНИЕ 2. Пусть $\beta \neq \sqrt{2} u T=2 q /(\pi p), q, p \in \mathbb{N}$. Тогда существует $\delta=\delta(T)>0$ такое, что для любого $v_{0}, 0<v_{0}<\delta$ уравнение (1) с граничными условиями (2) сильно устойчиво.

ЗАмЕчАниЕ 2. Для уравнения трубопровода (1) $0 \leq \beta \leq 1$.

ДокАЗАтЕльство. Считаем $v_{0}<\pi$. Оператор $P_{0}+v_{0}^{2} P_{1}$ положительно определен, поэтому $\sigma_{0}=\varnothing$ и присоединенные векторы отсутствуют. Разделим единичную окружность на $2 p$ дуг точками

$$
\mathfrak{M}=\left\{e^{i \frac{2 \pi}{2 p} s} \mid s=\overline{0,2 p-1}\right\}
$$

и занумеруем их, обходя против часовой стрелки начиная с 1 , числами от 1 до $2 p$. Имеем

$$
\sigma_{I}=\overline{\left\{\exp \left(i \lambda_{k} T\right) \mid \operatorname{Im} \lambda_{k}>0\right\}}, \quad \sigma_{I I}=\overline{\left\{\exp \left(-i \lambda_{k} T\right) \mid \operatorname{Im} \lambda_{k}<0\right\}} .
$$

При $v_{0}=0 \sigma(L)=\left\{ \pm(\pi n)^{2}\right\}_{n=1}^{\infty}$ и

$$
\sigma(U(T)) \subset\left\{e^{i 2 \pi \frac{k}{p}}, k=0, \ldots, p-1\right\}
$$

В силу (13) существует $\delta=\delta(T)$ такое, что при $0<v_{0}<\delta \sigma_{I}$ лежит на положительном расстоянии от $\mathfrak{M}$ на дугах с четными номерами (если $|\beta|<\sqrt{2}$ ) или с нечетньми (если $|\beta|>\sqrt{2}$ ). Так как множества $\sigma_{I}$ и $\sigma_{I I}$ комплексно сопряжены и количество дуг - четное число, получаем, что

$$
\sigma_{I} \cap \sigma_{I I}=\varnothing
$$

По теореме 2 уравнение (1) сильно устойчиво.

ЗАмЕчАниЕ 3 . В случае $\beta=0$ утверждение предложения 2 можно усилить: уравнение сильно устойчиво, если

$$
v_{0}^{2} \in\left(\frac{\pi^{2}(n-1)}{q}, \pi^{2}\left(\frac{n}{q}-\frac{n^{2}}{4 q^{2}}\right)\right), \quad 1 \leq n \leq 2 \sqrt{q}
$$

Автор выражает искреннюю благодарность своему научному руководителю професcopy А. Г. Костюченко за постановку задачи и постоянное внимание к работе 


\section{СПИСОК ЦИТИРОВАННОЙ ЛИТЕРАТУРЫ}

[1] Paidoussis M. P., Issid N. T. Dynamic stability of pipes conveying fluid // J. Sound and Vibration. 1974. V. 33. № 3. P. 267-294.

[2] Зефиров В.И., Колесов В. В., Милославский А. И. Исследование собственных частот прямолинейного трубопровода // Изв. АН СССР. Сер. Мех. тв. тела. 1985. № 1. С. 179-188.

[3] Shkalilov A. A. Operator pencils arising in elasticity and hydrodynamics: the instability index formula // Operator Theory: Advances and Applications. V. 87. Basel: Birkhäuser Verlag, 1996. P. 358-385.

[4] Милославский А. И. О спектре неустойчивости операторного пучка // Матем. заметки. 1991. Т. 49. № 4. С. $88-94$.

[5] Буй Та Лонг. Применение функциональных методов к некоторьм задачам устойчивости. Дисс. канд. физ.-мат. наук. М.: МГУ, 1988.

[6] Крейн С. Г., Петунин Ю. И. Шкалы банаховых пространств // УМН. 1966. Т. 21. № 2. C. $87-186$.

[7] Kato T. Linear evolution equation of hyperbolic type // J. Fac. Sci. Univ. Tokyo. Sect. I. A Math. 1970. V. 17. P. 241-258.

[8] Дергузов В.И., Якубович В.А. Существование решений линейных гамилтоновых уравнений с неограниченными операторами // Проблемы мат. анализа. Вып. 2. Линейные операторы и операторные уравнения, 1969.

[9] Дергузов В.И. Об устойчивости решений уравнений Гамильтна с неограниченными периодическими коэффиццентами // Матем. сб. 1964. Т. 63 (105). № 4. С. 591-619.

Московский государственный университет им. М. В. Ломоносова

Поступило

03.12 .1998

Исправленный вариант

30.08.1999 